\title{
USO DE FERRAMENTAS POR CEBUS APELLA (LINNAEUS) (PRIMATES, CEBIDAE) PARA OBTENÇÃO DE LARVAS DE COLEOPTERA QUE PARASITAM SEMENTES DE SYAGRUS ROMANZOFFIANUM (CHAM.) GLASSM. (ARECACEAE) ${ }^{1}$
}

\author{
Vlamir José Rocha ${ }^{2}$ \\ Nelio Roberto dos Reis ${ }^{3}$ \\ Margareth Lumy Sekiama ${ }^{2}$
}

\begin{abstract}
Tool use in Cebus apella (Linnaeus) (Primate, Cebidae) to get COLEOPTERA LARVAE THAT PARASITE SEEDS OF SYAGRUS ROMANZOFFLANUM (CHAM.) GLASSM. (ARECACEAE). This paper reports the behavior of the black capped capuchin monkey, Cebus apella, using tools to break seeds of Syagrus romanzoffianum in search of coleoptera larvae that live inside. The study area was the Parque Municipal Arthur Thomas ( $85,47 \mathrm{ha})$ located in the city of Londrina, Paraná state. In one year of study, using the ad libitum method, the monkeys were observed using rocks pretty much in the same way as if they were hammer and anvil. Individuals of both sexes including adults, subadults and juveniles with more than two years of age, were involved in such activity. The behavior of the animals consisted of they place the seeds on a large rock in the ground, and using a smaller rock which was hold among the hands, the seeds were beaten repeatedly until breaking. Then they ate the larvae and/or endosperm. The use of tools is thought to have brought benefits to the animals by allowing the access to a source of food that would not normally be used.

KEY WORDS. Primate, Cebidae, Cebus apella, behavior
\end{abstract}

O comportamento de utilizar ferramentas para obtenção de alimentos, é um fato conhecido em primatas. No Velho Mundo, chimpanzés, Pan troglodytes (Blumenbach, 1775), utilizam gravetos para "pescar" cupins e também usam galhos e rochas como martelo para quebrar frutos de casca dura (GOODALL 1965; vAN LAWICK-Godall 1971 apud IZAWA \& MizUNo 1977). Já em primatas do Novo Mundo, os primeiros registros relatando o uso de ferramentas pelas espécies do gênero Cebus em cativeiro datam da década de 1930 (KLÜVER 1933, 1937 apud ANDERSON 1990). Nas últimas décadas, foram realizados alguns trabalhos mais sistematizados testando a capacidade das espécies do gênero Cebus em usar ferramentas em cativeiro. A espécie mais estudada foi o macaco-prego, Cebus apella (Linnaeus, 1758), destacando-se os trabalhos de ViSALBERGHI \& ANTINUCCI (1986); ANTINUCCI \& VisAlBERGHI (1986); VisAlbERGHI (1987, 1990); RITCHIE \& FRAGASZY (1989); WESTERGAARD \& FRAGASZY (1987); ANDERSON (1990). Na

1) Contribuição número ??? do Departamento de Zoologia, Universidade Federal do Paraná.

2) Curso de Pós-graduação em Zoologia, Departamento de Zoologia, Universidade Federal do Paraná. Caixa Postal 19020, 81531-990 Curitiba, Paraná, Brasil.

3) Departamento de Biologia Animal e Vegetal, Universidade Estadual de Londrina. Caixa postal 6001, 86051-970 Londrina, Paraná, Brasil. 
natureza, FERNANDES (1991), trabalhando em área de manguezal, registrou indivíduos machos de C. apella utilizando pedaços de conchas de ostras, Crassostrea rhiziphorae (Guilding, 1828), para quebrar conchas inteiras. Além disso, IZAWA \& MizUNO (1977) e STRUHSAKER \& LELAND (1977) relataram que C. apella pode quebrar sementes e frutos de casca dura batendo-os um contra o outro ou contra troncos de árvores.

Neste trabalho, foi registrado na natureza o uso de rochas como martelo e bigorna por $C$. apella, para obtenção de larvas de Coleoptera que parasitam o interior de sementes da palmeira jerivá, Syagrus romanzoffianum (Cham.) Glassm.

\section{MATERIAL E MÉTODOS}

Um grupo de $C$. apella, composto de 44 indivíduos, foi estudado no período de outubro de 1993 a setembro de 1994 no Parque Municipal Arthur Thomas. O Parque está situado no perímetro urbano da cidade de Londrina (PR) entre as coordenadas de $23^{\circ} 15^{\prime}-23^{\circ} 30^{\prime} \mathrm{S}$ e $51^{\circ} 00^{\prime}-51^{\circ} 15^{\prime} \mathrm{W}$. Possui uma área de 85,47 ha dos quais cerca de 65 ha são representados por uma floresta alterada e heterogênea formando um complexo entrelaçado de ervas, cipós, arbustos e árvores, sendo denominada como floresta estacional semi-decidual. O restante da área compreende um viveiro de mudas, áreas de cultivos e um lago artificial. O relevo é acentuadamente íngreme podendo ultrapassar os $45^{\circ}$ de inclinação em algumas partes, chegando a possuir fragmentos de rochas expostas. O clima da região segundo a classificação de Köppen é do tipo cfa - clima subtropical, com temperatura média no mês mais frio inferior a $18^{\circ} \mathrm{C}$ e temperatura média no mês mais quente acima de $22^{\circ} \mathrm{C}$, com verões quentes, geadas pouco freqüentes e concentração das chuvas nos meses de verão, com tudo sem estação seca definida (IAPAR 1994).

O método utilizado para as observações dos animais foi o ad libitum (ALTMANN 1974) com auxílio de binóculo. A distância mínima de observação foi de cerca de três metros e, eram anotados, sempre que possível, o sexo e a idade aproximada. A determinação do sexo foi feito através da visualização das genitálias dos indivíduos e de características sexuais secundárias como formato de rosto, tamanho do topete e tamanho corporal. Para se determinar a idade aproximada, seguiu-se os mesmos critérios utilizados por IZAWA (1980). As rochas utilizadas pelos animais foram pesadas.

A palmeira S. romanzoffianum, cujos frutos e sementes são consumidos por C. apella, é uma espécie comum no local. Possui uma altura que varia de 5 a $10 \mathrm{~m}$ apresentando período de frutificação distribuído ao longo do ano com um pico entre o final do verão até metade do outono. Os frutos estão dispostos em cachos, têm cerca de $3 \mathrm{~cm}$ de comprimento e quando maduros, apresentam coloração laranjaamarelada. A semente pode apresentar em seu interior uma larva de uma espécie de Coleoptera (não identificada), com cerca de $1 \mathrm{~cm}$ de comprimento, que se alimenta do endosperma.

Para se determinar a porcentagem de sementes parasitadas pelas larvas, foram coletados ao acaso, 200 frutos maduros, de dois indivíduos de S. romanzoffianum. As sementes foram quebradas com auxílio de um martelo. 


\section{RESULTADOS}

O procedimento dos integrantes do grupo, consistia de se alimentarem da polpa madura do fruto de $S$. romanzoffianum, geralmente na própria palmeira, após, descartavam as sementes embaixo da planta-mãe. Posteriormente desciam ao solo e utilizavam as rochas para quebrar as sementes ou então, executavam esta tarefa após alguns dias. O comportamento realizado pelos macacos foi o de carregar as sementes na mão ou na boca até locais no solo onde encontravam uma rocha maior, que servia como bigorna, e uma menor, que servia como martelo. As sementes eram colocadas, uma por vez, sobre a rocha maior e, com a menor entre as duas mãos, os animais batiam repetidamente contra elas até quebrá-las (Fig. 1). Após, utilizavam os dedos ou os dentes caninos para terminar de abri-las, e então retiravam a larva de Coleoptera (Fig. 2). Quando essa larva não era encontrada, a semente era descartada ou o endosperma era consumido.

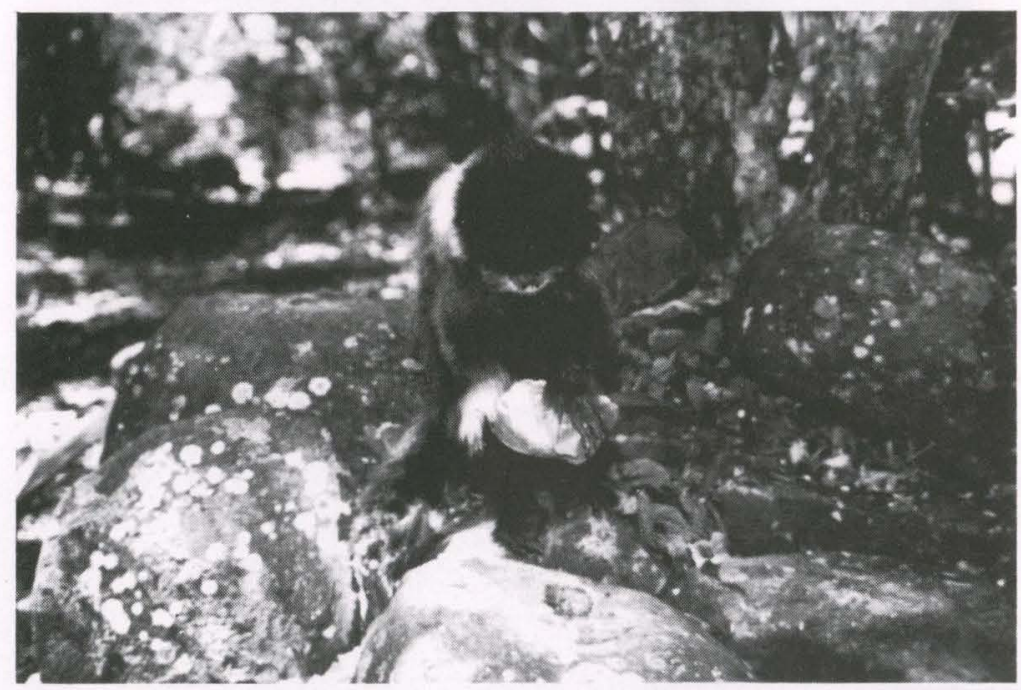

Fig. 1. Fêmea adulta de Cebus apella utilizando rochas como martelo e bigorna para quebrar sementes de Syagrus romanzoffianum.

Na maioria dos casos observados, os animais envolvidos eram de ambos os sexos e todos os adultos, subadultos e juvenis de cerca de dois anos de idade, exibiram o comportamento do uso de ferramentas. Contudo no caso dos juvenis, não foi constatado se todos os indivíduos realizavam tal comportamento. Indivíduos com menos de dois anos de idade, não foram observados utilizando ferramentas. Entretanto, em diversas ocasiões foi constatado que estes indivíduos e até mesmo juvenis mais velhos que sabiam utilizar as rochas como martelo e bigorna, permaneciam próximos de indivíduos aparentemente mais experientes que estavam utilizando ferramentas para tentar pegar as sementes quebradas que eram lançadas com o impacto da rocha (Fig. 3), atividade esta que eventualmente desencadeava interações agonísticas. 


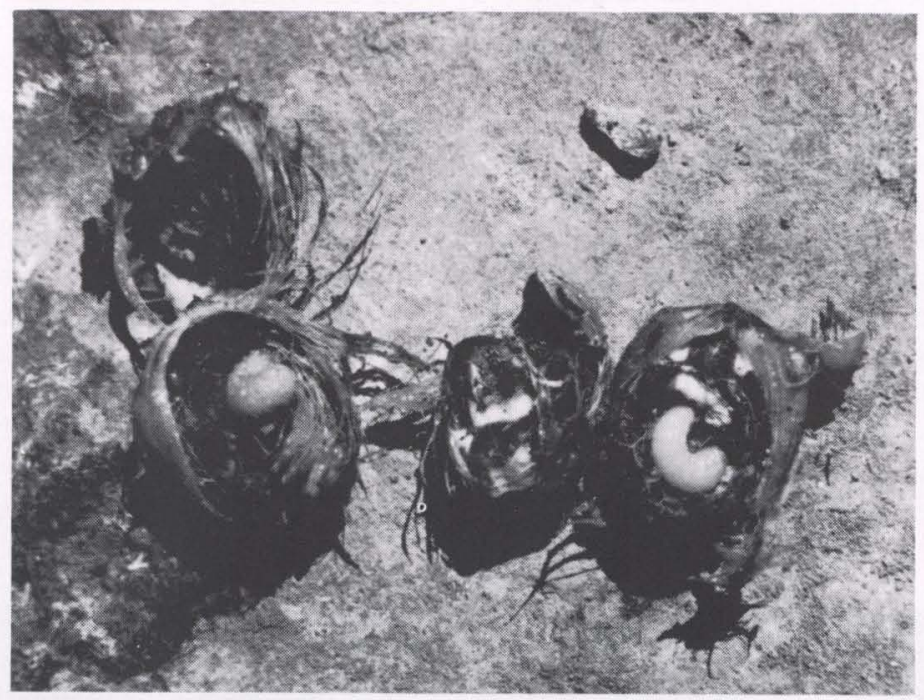

Fig. 2. Sementes de Syagrus romanzoffianum parasitadas por larvas de Coleoptera.

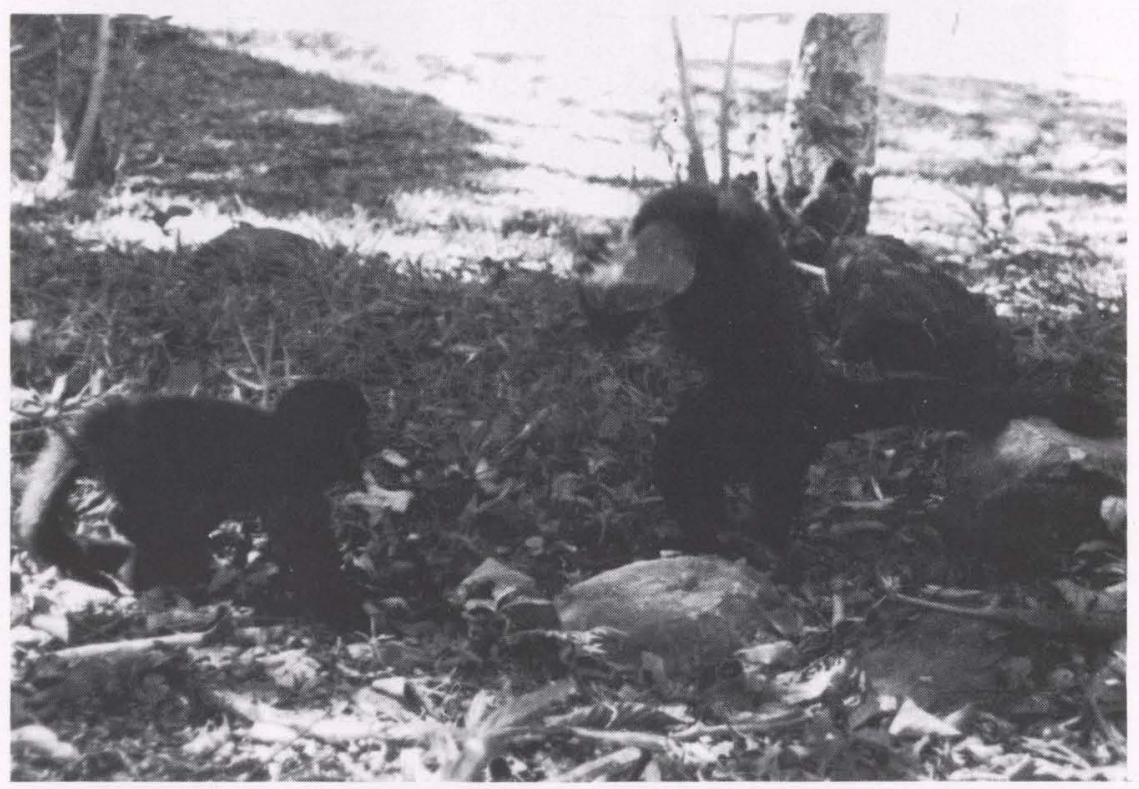

Fig. 3. Macho juvenil observando um macho adulto utilizando ferramentas.

Os animais adultos sempre tiveram maior êxito em quebrar as sementes, pois eram capazes de utilizar rochas mais pesadas que os animais mais novos, além de possuir mais experiência em realizar essa tarefa. $\mathrm{O}$ peso das rochas utilizadas pelos animais, variou de $200 \mathrm{~g}$ a $1000 \mathrm{~g}$. 
$\mathrm{O}$ índice de parasitismo das sementes pelas larvas de Coleoptera foi de $46 \%$ e todas as sementes parasitadas tiveram seu endosperma totalmente consumido.

\section{DISCUSSÃO}

Até o presente momento, na natureza, só havia sido registrado o comportamento de $C$. apella quebrando sementes ou frutos de casca dura, batendo-os um contra o outro ou contra troncos de árvores (IZAWA \& MIZUNO 1977; STRUHSAKER \& LELAND 1977). ViSALBERGHI (1990) sugere que, devido ao estilo de vida arborícola de $C$. apella, é improvável que na natureza, esses animais tenham contato com situações que favoreçam o uso de rochas como martelo e bigorna. Porém, no presente estudo encontrou-se situações que favoreceram o uso dessas ferramentas por esses primatas, tais como: presença de rochas de tamanho adequado para serem utilizadas como martelo e bigorna; presença das sementes de S. romanzoffianum de interesse alimentar para C. apella; e ausência de predadores na área de estudo, o que proporcionou aos animais um maior tempo de permanência em atividades no solo.

Comportamento semelhante ao descrito para $C$. apella, no Parque Arthur Thomas, foi registrado por ANDERSON (1990) em situações experimentais em cativeiro, o que demonstra que esta espécie possui grandes habilidades motoras, podendo realizar este comportamento sempre que ocorrerem situações favoráveis no ambiente.

A constatação de que apenas indivíduos de dois anos ou mais possam utilizar ferramentas, provavelmente está relacionado com o tempo requerido para o aprendizado, pois esse comportamento requer força e uma refinada coordenação motora para que os animais tenham êxito em quebrar as sementes. ANDERSON (1990) também registrou jovens de dois anos de idade utilizando ferramentas para quebrar sementes de Prunus amygdalus, Corylus avellana e Juglans regia.

Levando em consideração que o peso corporal de um animal adulto, segundo Silva (1994) pode variar de $2 \mathrm{Kg}$ a $4 \mathrm{Kg}$, as rochas utilizadas como martelo, podem pesar entre $1 / 2$ a $1 / 4$ do corpo de um animal adulto. Assim, o retorno energético obtido pelos primatas ao consumir o parasito ou o endosperma, provavelmente, deverá ser maior do que o gasto de energia requerido para utilizar as rochas como ferramentas.

Outro fato a ser considerado é que as larvas de Coleoptera ao consumirem o endosperma, tornavam as sementes parcialmente ocas, e portanto, mais fáceis de serem quebradas do que aquelas que não estavam parasitadas, diminuindo o esforço realizado pelos animais.

O comportamento apresentado por $C$. apella foi provavelmente adaptativo às condições encontradas em seu habitat e pode ser comparado ao comportamento de utilizar ferramentas apresentado por Pan troglodytes, descrito por GOODALL (1965), VAN LAWICK-GOODALL (1971) apud IZAWA \& MizUNO (1977).

O fato descrito acima, ainda não foi observado em outros grupos de C. apella que ocorrem na região de Londrina, provavelmente pela ausência de algum dos fatores que favoreçam o uso de ferramentas. Entretanto foi observado o comportamento de indivíduos baterem frutos, galhos, rochas e objetos contra troncos de árvores ou contra o solo, o que demonstra a habilidade motora que estes animais também teriam em utilizar rochas como martelo e bigorna. 
A capacidade que um grupo de primatas possui em usar ferramentas em uma área e em outras não, é denominado por IZAWA \& MiZUNO (1977) e KAWAI (1965) apud IZAWA \& MizUNO (1977), como sendo uma pré-cultura, adquirida devido às situações do ambiente, o que corrobora com as observações realizadas para o grupo de $C$. apella que vivem no Parque Municipal Arthur Thomas.

Deste modo, pode-se afirmar que o uso de ferramentas por Cebus apella trouxe benefícios aos animais, pois permitiu o acesso a uma fonte de alimento que normalmente não seria utilizada.

\section{REFERÊNCIAS BIBLIOGRÁFICAS}

ALTMANN, J. 1974. Observational study of behavior sampling methods. Behaviour 49: 227-267.

ANDERSON, J.R. 1990. Use of objets as hammers to open nuts by capuchin monkeys (Cebus apella). Folia Primatol. 54: 138-145.

ANTINUCCI, F. \& VisAlbERGHI, E. 1986. Tool use in Cebus apella: A case study. Int. Jour. Primatol. 7: 351-363.

FERNANDES, M.E.B. 1991. Tool use and predation of oysters (Crassostrea rhizophorae) by the tufted capuchin, Cebus apella, in brackish water mangrove swamp. Primates 32 (4): 529-531.

Goodall, J. 1965. Chimpanzees of the Gombe Stream Reserve, p.425-473. In: I. DEVORE (Ed.). Primate behavior. New York, Holt Rinehart \& Winston Inc., $654 \mathrm{p}$.

IAPAR. 1994. Cartas climáticas do estado do Paraná 1994. Londrina, Iapar, 49p. IZAWA, K. 1980. Social Behavior of the black-capped capuchin (Cebus apella). Primates 21 (4): 443-467.

IZAWA, K \& A. MIZUNO. 1977. Palm fruit cracking behavior of wild black-capped capuchin (Cebus apella). Primates 18: 773-792.

RITCHIE, B. \& D.M. FragASZY. 1989. A capuchin monkey (Cebus apella) use tools on her infant's wound. Amer. Jour. Primatol. 16: 345-348.

Silva, F. 1994. Mamíferos silvestres - Rio Grande do Sul. Porto Alegre, Fundação Zoobotânica do Rio Grande do Sul, 246p.

StruHSAKer, T.T. \& L. Leland. 1977. Palm-nut smashing by Cebus apella in Colombia. Biotrópica 9; (2) 124-126.

VISALBERGHI, E. 1987. Acquisition of nut-cracking behaviour by 2 capuchin monkeys (Cebus apella). Folia Primatol. 49: 168-181. 1990. Tool use in Cebus. Folia Primatol. 54: 146-154.

Visalberghi, E. \& F. AntinUCCI. 1986. Tool use in the exploitation of food resources in Cebus apella, p.57-62. In: J.G. ELSE \& P.C. LEE (Eds). Primate Ecology and Conservation. Cambridge, Cambridge University Press, 393p.

WestergaARD, G.C. \& D.M. Fragaszy. 1987. The manufacture and use of tools by capuchin monkeys (Cebus apella). Jour. Comp. Psychl. 101: 159-168.

Recebido em 04.VII.1997; aceito em 03.XI.1998. 